\title{
LVIII.-Ozone from Pure Oxygen: its Production and its Action on Mercury, with a Note on the Silent Discharge of Electricity.
}

\section{By W. A. Shenstone, Lecturer on Chemistry in Clifton College, and J. Tudor Cundall.}

For the purposes of some experiments we are making with the hope of obtaining further information concerning the influence of the silent discharge of electricity on oxygen and other gases, we have had occasion to prepare oxygen gas of a high degree of purity and to transfer it to experimental apparatus of various kinds without contamination. We have taken the opportunity which has thus occurred to make the experiments that we now have the honour of describing to the Chemical Society.

In their classical research, "On the Volumetric Relations of Ozone," Andrews and Tait (Phil. Trans., 1860, Part I, p. 113) have already shown that ozone may be produced from oxygen of considerable purity. They express their opinion that not more than one part of nitrogen was present in every thousand parts of the oxygen they employed, and, though they do not describe any analyses in support of that opinion, there can be no doubt that the gas employed was more than sufficiently pure for the object they had before them, viz., to establish the elementary nature of ozone. But, taking into consideration the fact that unprotected india-rubber connections were employed in some parts of their apparatus, and that they dried the gas merely by passing it over fragments of glass moistened with sul1huric acid, it seems improbable that they operated upon oxygen free, in the highest attainable degree, from nitrogen and moisture. The experiments described by Brodie (ibid., 1872), although also made with great care and delicacy, probably were not made with oxygen of the highest attainable purity. In consequence of the interest which now attaches to the chemical behaviour of substances in an extremely pure state, it seemed desirable to repeat experiments 
like those of Andrews and Tait with all the additional precautions that have become possible.

Whilst we were still engaged in constructing the apparatus presently to be described, a paper on the production of ozone, by Professors J. J. Thomson and R. Threlfall was read before the Royal Society (Proc. Roy. Soc., 1886, 40, 340). In this paper, it is stated that ozone is formed in large quantities when an electric spark passes through dry oxygen. In the experiment described, however, air was employed, not oxygen, and no details are given in the paper of the mode of recognising the ozone, of the quantity produced, or of the behaviour of dry ozone to any other substance in a dry state; therefore we considered that the subject still deserved careful examination.

\section{The Preparation and Storage of Oxygen.}

After constructing and trying various apparatus for this purpose, we adopted that which was described at the Birmingham Meeting of the British Association (Brit. Ass. Report, 1886), by the aid of which two of the specimens of oxygen used for our experiments were prepared. But further experience in constructing glass apparatus on a large scale has enabled us now to adopt a modification of that apparatus, which affords a far more complete guarantee of the purity of a gas prepared within it after it has been stored for considerable periods of time than was the case with the original simpler arrangement.

In dealing with gases containing less than one part of impurity per thousand, the mode of preparing and storing the gas affords an indication of its quality which is at least as important as any evidence that can be derived from analysis. This consideration will excuse us if we seem to devote a disproportionate part of our paper to a description of the apparatus we now employ.

The general form of the apparatus is shown in Fig. 1. All its parts have been united by welding in the blowpipe flame, except that the sampling tube $K$ is attached by a ground joint at $\mathrm{L}$, which is protected by a cup of mercury with a layer of almond oil above it. The Sprengel pumps attached to $\mathrm{C}$ and $\mathrm{D}$ also are not only welded to the main apparatus, but were themselves entirely put together by blown joints. The taps employed are those known in this country as Cetti's patent, air is prevented from entering the apparatus by cups of mercury and oil as shown in the diagram. Further, the mercury which is introduced into various parts of the apparatus from time to time passes in all cases through efficient air-traps. It appears, therefore, to be impossible for air to gain admittance to any gas that may be stored within 
the apparatus so long as no part of the glass work is broken. As a rule, we find that air-traps such as G (Fig. 1) answer very well, but occasionally air collects in a trap with inconvenient rapidity, and we think the trap shown at $\mathrm{L}$ is more satisfactory. In this form of trap, which we do not remember to have previously seen described, there is attached at the shoulder a narrow tube M, drawn out at its upper end. When air has collected at L, it can be expelled, without admitting air to the store of gas within the apparatus, by opening the tip of $\mathrm{M}$ and raising the reservoir of mercury attached to the india-rubber tube $\mathrm{N}$.

Fig. 1.

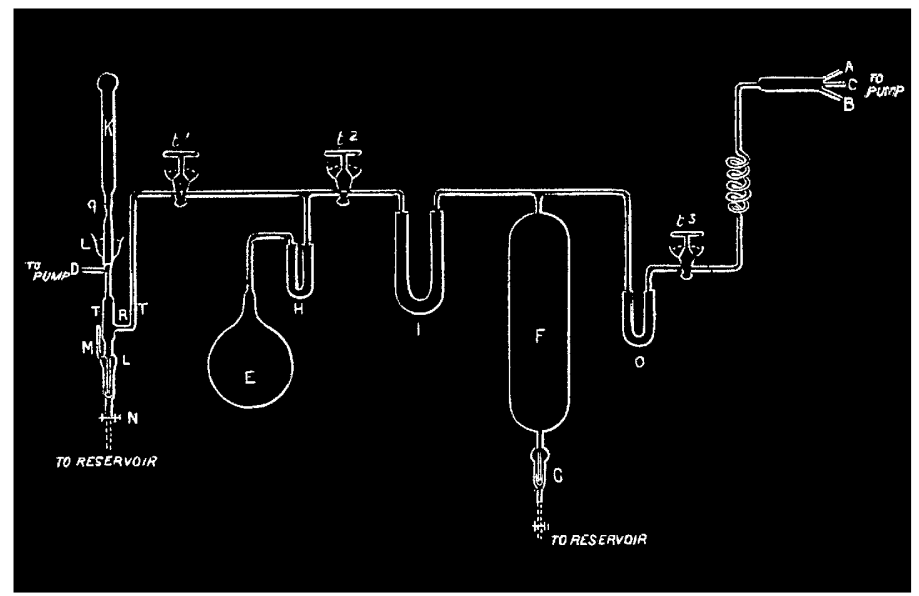

For generating oxygen, $\mathrm{E}$ is charged with a mixture of the chlorates of sodium and potassium in molecular proportions. This is prepared by fusing the two salts together, and powdering the mixture after it has solidified on cooling. We find this mixture a very convenient source of oxygen on account of its low melting point, provided care be taken to employ sodium chlorate that is perfectly free from the tartrate.

To fill the apparatus in the first instance, $\mathrm{F}$ is filled with mercury from a reservoir not shown in the diagram, the taps $t^{1}, t^{2}$, $t^{3}$ are opened, and the apparatus is well exhausted by the Sprengel pumps attached to $\mathrm{C}$ and $\mathrm{D}$. Oxygen is then generated in $\mathrm{E}$ until the whole apparatus, including $\mathrm{F}$, is filled. It is then re-exhausted, refilled, and left with its charge of oxygen for several days in order that any nitrogen adherent to the glass may, as far as possible, be removed by the molecular impacts of the oxygen; lastly this charge of gas 
is also removed, and the final charge is generated from the chlorates in E. A simple calculation shows that if the pumping apparatus only removes $\frac{759}{760}$ of the contained gas at each operation the amount of nitrogen present in the oxygen thus prepared will be about $\frac{100000000}{4000}$ of the whole. After the apparatus has been once charged, when the supply of oxygen remaining in $\mathrm{F}$ becomes small, $t^{2}$ is closed, $t^{1}$ is opened, and $\mathrm{E}$ is heated as before. The first portions of oxygen evolved are permitted to escape by D through the fall tube of the Sprengel pump, and so soon as all fear of accidental admission of air in consequence of the breaking of $\mathrm{E}$ is past, $t^{1}$ is closed, $t^{2}$ opened, and F filled as before. The taps have been very carefully reground, and we find that they resist the passage of air excellently, so that in case of $\mathrm{E}$ breaking we believe it might be replaced and its contained air removed without any sensible contamination of the gas ready for use in F. As we have been fortunate in our selection of flasks, however, we have not had occasion to put this opinion to the test of an experiment. The tube $\mathrm{H}$ contains glass wool which filters out suspended particles of salt from the gas. $\mathrm{I}$ is a large $\mathrm{U}$-tube containing solid potash to absorb any minute trace of carbon dioxide that might accidentally be evolved in consequence of the presence of carbonaceous particles in E. As we adopt every possible means of avoiding this in preparing the chlorates, and as the chlorates in $\mathrm{E}$ are kept in a fused condition for a considerable period before $\mathrm{E}$ is tixed in its place, the necessity of $I$ is doubtful.

We have repeatedly examined considerable quantities of our gas for such impurities as carbon dioxide and chlorine, but have never obtained any indication of their presence.

$\mathrm{O}$ contains phosphorus pentoxide, but the employment of this substance is not related to the experiments we are about to describe. Various pieces of apparatus have been attached to $A$ and $B$ temporarily or permanently as was required.

\section{Testing the Purity of the Gas.}

As we have remarked above, there is a strong presumption that the proportion of nitrogen present in oxygen prepared as we have described it is so small as to be far beyond measurement. We have thought it right, however, so far as possible, to test the truth of this assumption.

Specimens of gas for examination may be obtained from time to time in sampling tubes, such as $K$, capable of holding about 30 c.c. of the gas. Our method of tilling them has been to let the gas be free to diffuse throughout all parts of the apparatus for many hours, then to close $t^{\mathrm{I}}$, and after slightly reducing the pressure in $\mathrm{K}$ by the pump 
attached to $\mathrm{D}$, to carefully seal off $\mathrm{K}$ at $q$, where the glass is well thickened beforehand, in order that it may not collapse irreguiarly when softened. A fresh tube is afterwards put in the place of $K$, by filling the trap $\mathrm{R}$ to the level TT with mercury, removing the stopper from L, welding to it another tube, replacing the stopper in $\mathrm{L}$, and exhausting and filling this tube with oxygen as previously described, the mercury in the trap serving to protect the stock of gas from contamination meanwhile. We also attach various pieces of apparatus that we require to fill with oxygen by means of $\mathrm{L}$.

The tube $k$ containing the sample of oxygen is placed with its thin expanded end downwards in a glass vessel of mercury, A, which has been previously thoroughly freed from air bubbles, heated on a sand-bath, and then allowed to cool (Fig. 2). The thin glass is easily broken by pressing it on the bottom of $\mathrm{A}$, and a little mercury

Fig. 2.

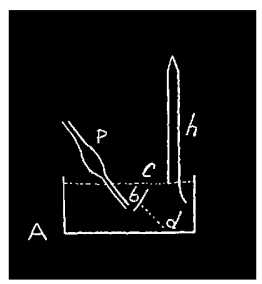

enters the tube. Reagents are then introduced. For some time, we had great difficulty in securing a suitable reagent for our purpose; the best of those ordinarily employed, even when prepared with the greatest care, left a residue which seemed to indicate the presence of about one part of nitrogen in one thousand parts of the gas. After several failures, it occurred to us that phosphorus, though in most cases an unsatisfactory absorbent of oxygen, would not be unsuitable in the case of pure or nearly pure oxygen. In using phosphorus, however, we find that the greatest care must be taken not to permit moisture to accompany it, because the presence of a very small quantity of water results in the production of a sensible volume of a fixed gas, which we are inclined to suppose to be phosphine. The method which has given us the best results is as follows:-Melted phosphorus is drawn into a pipette $P$, which has a very long and rather wide end at $b$. It is then boiled for some time to expel moisture, and the end $b$ is sealed at $\mathbf{C}$. The basin of mercury in which $k$ has been opened is then placed upon a tripod support, the pipette $\mathrm{P}$ brought into the position shown in Fig. 2, and its end broken off at $\vec{d}$ without exposing the phosphorus to the air; the tip of the pipette is then brought 
under the mouth of $k$, and the mereury is warmed till the phosphorus melts ; a little of it is then forced out of the pipette. Heat is cautiously applied to $k$ till the phosphorus is ignited, if that does not at once occur. Finally $k$ is heated so that vapour of phosphorus fills the space previonsly occupied by oxygen. The whole is then allowed to cool, the extremely minute space occupied by the residual gas noted, and its rolume determined by filling it with water which is afterwards weighed. As the volumes to be measured are very small, the results can only be regarded as close approximations. If the phosphorus employed has not been thoroughly dried by boiling, not only is the volume of residue somewhat greater, but we find that sometimes it may be increased by the prolonged action of heat.

A portion of the oxygen we employed in the second pair of experiments now described was tested in this manner. It left a residue amounting to no more than one part in five thousand of the gas. Taking into consideration the very great difficulty of obtaining phosphorus perfectly free from air, moisture, and phosphorous acid, we are not inclined to consider that this result indicates that only $\frac{4999}{5000}$ of our gas was oxygen, but rather that the purity of our oxygen considerably exceeds this.

\section{Firs:3.}

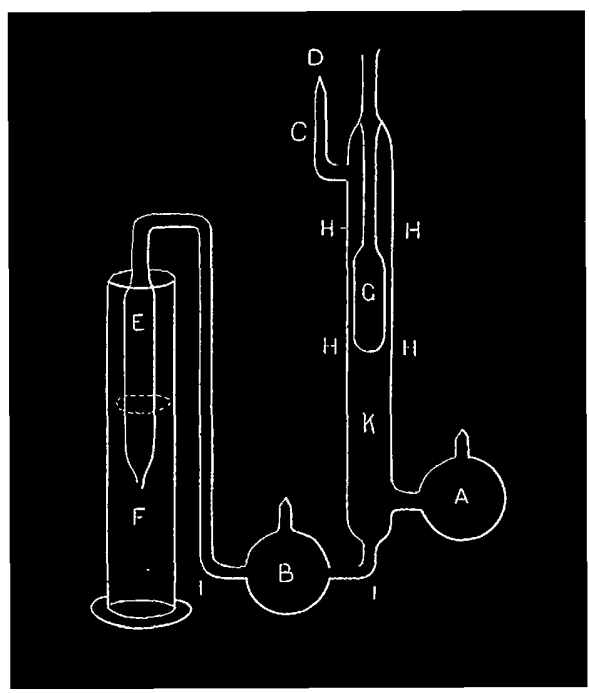

Our first pair of experiments were raade on oxygen collected in tubes such as that shown in Fig. 3; they were filled from the 
apparatus described at the Birmingham meeting of the British Association (loc. cit.). Analysis showed that the gas when introduced into them contained not less than 99.96 per cent. of oxygen, and probably a considerably greater proportion. Although sereral india-rubber connections were used in that apparatus, they were all well protected from the outside air by mercury, and exposed to a vacuum for many hours before any gas was collected in the apparatus, whereby ample opportunity was afforded for the escape and removal of any occluded gas.

The globes A, B were filled with phosphorus pentoxide, and the two pieces of apparatus were filled with oxygen through $\mathrm{C}$ and sealed off at D; in filling them, all the precautions described in connection with the preparation of oxygen were taken. The tubes were then placed in a moderately warm cupboard from June 9 th, 1886 , to March 31st, 1887. The end of the arm $\mathrm{E}$ was then broken under the surface of sulphuric acid in the vessel $F$, the position of the sulphuric acid in $\mathrm{E}$ noted, and the gas electrified by connecting dilute sulphuric acid in $\mathrm{G}$, and a tinfoil envelope at $\mathrm{HH}$ with the electrodes of a Rhumkorf coil capable of giving a spark of 4 or 5 inches. As $B$ was closely packed with phosphorus pentoxide in a fine powdery condition, diffusion across it would occur very slowly, and we believe that any minute traces of moisture that might be communicated by the sulphuric acid to the oxygen would scarcely be permitted to pass through B. If we are wrong on this point, however, it will rather give emphasis to our results than detract from their value, inasmuch as we believe the yield of ozone in our experiments with these tabes is decidedly high for the temperature at which the experiments were made. Moreover, the results of a second series of experiments in which our gas was not brought into contact with sulphuric acid or any other possible source of moisture confirm our general result. Under the influence of the electric discharge, a steady diminution of the volume of the gas set in, which continued for a long period of time; doubtless in consequence of the slowness of diffusion across the bulb B. After frequently repeated electrification for short periods during 24 hours, the new position of the sulphuric acid in $\mathrm{E}$ was noted, the apparatus cut into three parts at I, I, and the volumes required ascertained in the usual manner. In one experiment, an unfortunate accident to $\mathrm{E}$ compelled us to do no more than estimate the contraction that occurred. We were very careful to avoid overestimation, and came to the conclusion that a contraction corresponding to the conversion into ozone of at least 7.5 per cent. of the oxygen employed had occurred, although the action of the electricity was still incomplete. In a subsequent observation, we learnt, however, that the contraction of oxygen when electrified in contact with sul- 
phuric acid must not be depended on for obtaining an exact measure of the ozone formed, unless certain precautions, which we omitted in this case, are taken. The second tube was treated like the first, except that the gas was electrified at intervals for three days.* The discharge in this tube was, we believe, singularly free from the larger sparks of electricity which reveal their presence by the sound which they produce, and which often accompany the so-called silent discharge. It was, in fact, more nearly a silent discharge than anything we had previously met with. Although the temperature at which we worked was never lower than $10^{\circ} \mathrm{C}$., the contraction that occurred was so great as to indicate the conversion of about 15 per cent. of the oxygen into ozone. This led us to doubt the entire trustworthiness of this method of measuring the formation of ozone, which we had copied from Andrews and Tait. And we therefore attached a burette containing turpentine to $\mathrm{C}$ by means of an india-rubber tube filled with turpentine and closed by a clamp. Then the end having been broken we allowed a small known quantity of turpentine to flow over the inside of $K$. The absorption was complete in about an hour, and amounted to $10 \cdot 25$ c.c. The volume of the residual oxygen under the same conditions of temperature and pressure was $116 * 2$ c.c. (the $\mathrm{P}_{2} \mathrm{O}_{5}$ being neglected), from which we find that the amount of oxygen converted into ozone was $11 \cdot 7$ per cent. of the quantity of oxygen employed in the experiment (a little higher indeed as the volume occupied by the $\mathrm{P}_{2} \mathrm{O}_{5}$ has been added to the true volume of residual oxygen present). This yield of ozone is high, as no precautions could be taken to electrify the oxygen at a low temperature. This considerably exceeds what is ordinarily produced by the passage of a current of oxygen through a similar apparatus of the Siemens type, our experience being that under similar conditions as to temperature a yield which exceeds 6 per cent. is satisfactory. It is, however, very difficalt to obtain concordant results in the production of ozone at different or indeed at similar temperatures. It does not, however, reach the proportion-25 per cent.-which would correspond to the contraction observed by Andrews and Tait in one of their experiments, in which a different mode of electrification was employed. In their experiments, however, oxygen was ozonised in contact with sulphuric acid, and the results, therefore, are almost certainly somewhat high. We have now prepared some tubes of oxygen provided with means of electrification similar to those employed by these observers, and therefore in the course of six montbs

* The actual period during which the discharge took place considerably exceeded an hour. The temperature of the air at the time of the experiment varied from $10^{\circ}$ to $11^{\circ}$. That of the tube at $\mathrm{H}-\mathrm{H}$ was rather higher. 
hope to be able to report whether an increased yield of ozone results in this case from the employment of pure and dry oxygen.

3. When the apparatus for storing oxygen, described in the earlier part of this paper, was complete, we filled two tubes like that shown in Fig. 4 with oxygen, a sample of which taken at the same time and

FIG. 4.

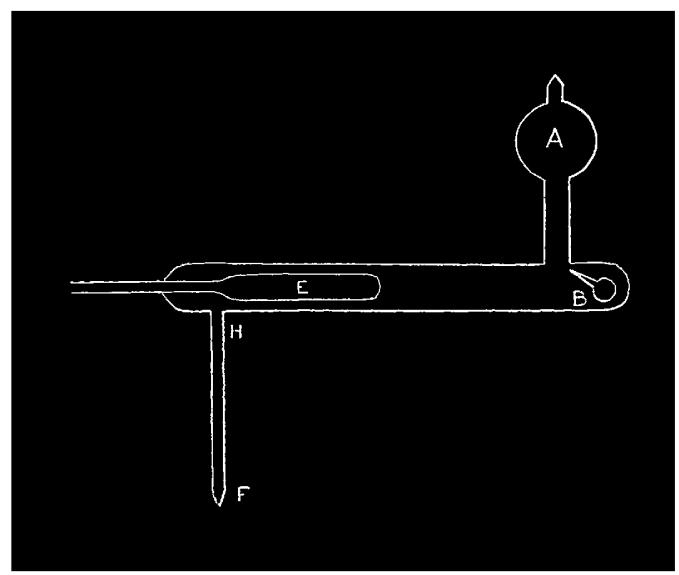

examined with phosphorus was found to yield only $\frac{1}{5 \frac{1}{0} 00}$ of residue, and which for the reasons already given we regard as in all probability containing a very considerably smaller proportion of nitrogen than this. $\mathrm{A}$ is a chamber containing phosphorus pentoxide, $\mathrm{B}$ a small bulb of pure mercury containing neither air nor moisture. One of these tubes filled with the purest oxygen we have yet obtained was kept for seven weeks; the gas within it was then electrified by connecting the poles of the coil with dilute sulphuric acid in $\mathrm{E}$, and tinfoil fixed around the tube from $\mathrm{H}$ to $\mathrm{H}$. Afterwards, the bulb $\mathrm{B}$ containing the mercury was broken. So soon as this was done, the surface of the mercury lost its convexity, and the mercury adhered to glass in the well-known manner to such an extent that it was easy to attach the remains of the glass bulb to any part of the tube to which mercury adhered. After 24 hours, however, none of the usual signs of oxidation were apparent on the mercury, and after a few days it showed signs of recovering its ordinary condition. The end $F$ of the tube was then attached (by india-rubber) to a wider tube containing paper moistened with potassium iodide and starch solution, its tip broken off, and some of the gas pumped quickly over the test-paper, but no evidence of the presence of ozone was obtained in this way, nor had the gas that remained in the tube any odour of 
ozone when we afterwards examined it. It is true that the indiarubber connection employed in this case is not entirely satisfactory, but no other suggested itself, and we took care that the gas should rush quickly through it into a partly exhausted space, where it had time to act upon the test employed. We cut up the tube and examined the residual mercury, and found that it could readily be run into perfectly clean globules by rubbing together the detached portions with a fragment of wood. This leads us to conclude that perfectly dry ozone is completely decomposed by mercury without the mercury undergoing permanent oxidation. This conclusion is confirmed by subsequent experiments.

It is well known that the distinctive action of ozone on mercury is accompanied by very evident oxidation, and we find that mercury is only capable of thus destroying ozone without itself undergoing some degree of oxidation when the ozone is in a very dry condition. We have passed oxygen generated from chlorate of potassium, and purified by moist and dry caustic potash in a slow stream through an ozone generator, then over a column of phosphorus pentoxide 11 inches long, and then through pare mercury placed in a small washing tube, all air having been previonsly driven from the apparatus by the passage of a very long-continned stream of oxygen, and have found that on sealing the tube with its contained ozonised oxygen, although scarcely any signs of oxidation appeared after an hour, very decided indications of oxidation developed after 12 hours. Andrews and Tait found that when bulbs of mercury are broken in vessels containing ozonised oxygen, the mercury is oxidised, and an expansion consequent upon the destruction of ozone by the mercury also occurs which, however, never restores the gas to quite its original volume. In the most favourable cases, a permanent contraction, amounting to one-sixth of the contraction on ozonisation, remained. This may have been due to oxidation of the mercury alone or partly also to absorption of ozone by the sulphuric acid employed in the pressuregauge. In order to confirm or disprove our conclusion, that in our experiment the ozone was entirely destroyed without oxidation of the mercury, we used our second tube of purest oxygen for the modification of the experiment which follows. After the oxygen had been submitted to the drying action of the phosphorus pentoxide in $\mathrm{A}$ for eight weeks, the end of the side tube F (Fig. 5) was immersed in mercury that had just before been strongly heated and placed while still warm in the tube G. The tip of $F$ was broken off by pressing it against the sides of $G$, and $F$ was fixed with a cork in such a position that $\mathrm{G}$ formed an air-trap to prevent the entrance of air from $H$ into the pure oxygen. To $G$ an adjustible reservoir $S$ containing mercury was attached. By means of the reservoir this gas in B was 
FIG. 5.

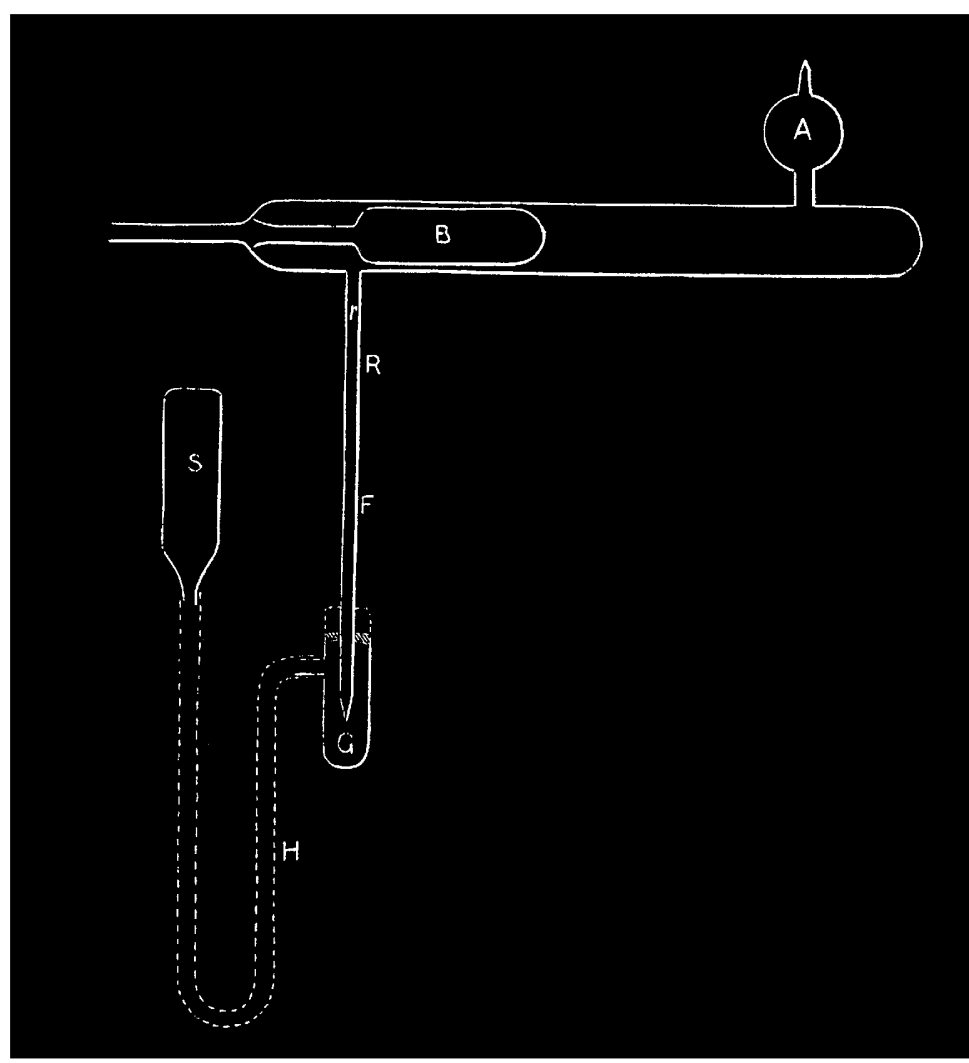

brought to a reference $\operatorname{mark} R$, and its pressure, viz., $769.8 \mathrm{~mm}$, and the temperature of the air $\left(12 \cdot 3^{\circ}\right)$ were observed. The gas was then electrified as before (the level of the mercury in F being slightly reduced). The production of ozone in this case quickly approached the maximum owing, perhaps, partly to its destruction by the mercury in $\mathbf{F}$, but chiefly to a decreased rate of production in consequence of the rise of temperature that occurred about $B$; for it was not possible to adopt the mode of repeated brief periods of electrification with considerable periods of subsequent cooling, that we employed in the previous experiments, in consequence of the destruction of ozone by the mercury that would have occurred during the periods of delay. After electrification, the pressure required to bring the gas to the mark $R$ was again observed, and was found to be $754.1 \mathrm{~mm}$, a diminution that corresponds to the presence of $6 \cdot 1$ per cent. of ozone in the gas. 
As the ozonisation was towards the end conducted at a rather high temperature, and as destruction of the ozone by the mercury must have occurred during the progress of the experiment, and during the cooling down of the tube after the electrification was stopped, this may be regarded as a very good yield of ozone.

The ozonised oxygen was left for twenty-four hours in contact with the mercury in $\mathrm{F}$, which was then repeatedly brought into view by adjusting the position of the reservoir $\mathrm{S}$. We were unable to perceive any signs of oxidation of the mercury even after several days had elapsed.

Although by this time it was no longer possible to observe the mercury in $F$ at the line $R$ (in consequence of the adhesion of the mercury to the glass), and so to ascertain at what pressure the oxygen in the apparatus would now occupy its original volume at $12 \cdot 3^{\circ} \mathrm{C}$. we succeeded in getting a close approximation to this in the following manner :-

Having succeeded in getting the mercury visible at a point near or a little above $r$, the pressure at which the gas occupied this new volume was observed. It was found to be $774 \cdot 2 \mathrm{~mm}$. when corrected for difference of temperature. A subsequent experiment made, after cleaning the tube, showed that to compress air from $\mathrm{R}$ to $r$, a pressure of $5 \cdot 4 \mathrm{~mm}$. was required: hence $774 \cdot 2-5 \cdot 4=768 \cdot 8$ was the pressure at which the gas would now fill our tube to the reference mark $R$. As the pressure at which it originally occupied the same volume was $769.8 \mathrm{~mm}$., it appears that of the contraction observed on ozonification (which corresponded to a diminution of pressure of $15.7 \mathrm{~mm}$.), all but so much as corresponds to a pressure of $1 \mathrm{~mm}$. had been restored by the action of the mercury; that is to say, $\frac{2}{16}$ only of the contraction was permanent. When the difficulties under which these measurements were taken are considered, this seems to us to strongly confirm the conclusion we had come to from our examination of the appearance of the mercury in two separate experiments, viz., that when dry ozone is decomposed under the influence of dry mercury, no oxidation of the metal takes place. Andrews observed that the permanent contraction of oxygen which had been ozonised and subsequently treated with mercury was greater in proportion to the suddenness with which the mercury acted. In our first experiment, by disturbing the metal with the fragments of glass, we did our best to hasten the change. In our second experiment, however, it was not possible to do otherwise than permit the change to proceed slowly.

From the experiments we have made we draw the following conclusions :-

1. That pure and dry oxygen is partly converted into ozone by the action of the silent electric discharge. 
2. That when ozone generators of the Siemens type are employed, the proportion of oxygen converted into ozone in the case of pure and dry oxygen appears to be somewhat greater than in the case of less pure oxygen under otherwise similar circumstances.*

3. That dry ozone is completely destroyed by contact with mercury, after some hours, without pereeptible oxidation of the mercury, such as occurs to a greater or less extent when mercury and imperfectly dry ozone come into contact.

\section{Note on the Silent Discharge of Electricity.}

The influence of purity and dryness on the formation of ozone from oxygen by silent discharge may be due to one or both of two independent causes.

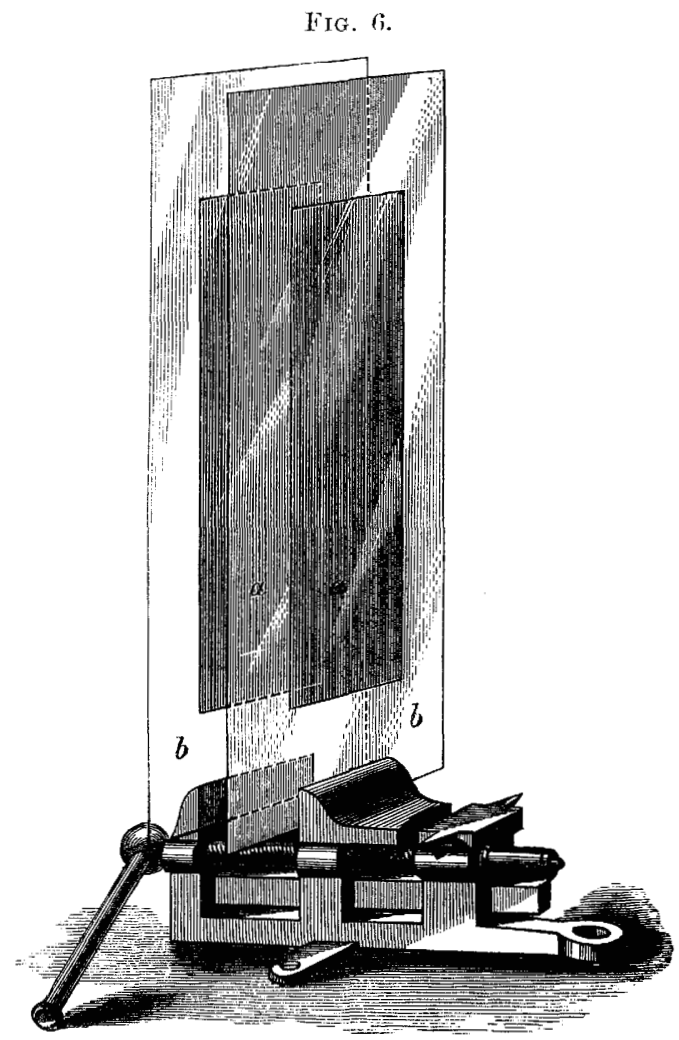

* This, howerer, is based chiefiy on our own experience, and it is diffeult to compare our work with that of others until the influence of temperature and other circumstances on the production of ozone is better understood than at present. 
a. Andrews and Tait (loc. cit.) have shown that, if the conditions be such as to favour the combination of nitrogen and oxygen, the presence of nitrogen in oxygen is unfavourable to the production of ozone, in consequence of the mutaal action of ozone and peroxide of nitrogen. Indeed, they state that if a mixture of I part of nitrogen and 499 parts of oxygen be submitted to the action of a few large sparks of electricity, the mixture yields no ozone when subsequently submitted to a glow discharge. As under ordinary conditions the discharge in ozone generators of the Siemens type is apt to consist partly of rather large sparks which may not improbably lead to the production of peroxide of nitrogen, ${ }^{*}$ it is evident that the complete absence of nitrogen is likely to be favourable to the production of ozone from oxygen in induction tubes.

$b$. The dryness of the oxygen, and of the surfaces of the tubes between which the discharge takes place, may affect the character of the discharge within it. In connection with this, the following observation will be interesting:-

In order to get an idea of the character of the electric discharge as it occurs in an ozone generator, we have repeated an experiment shown by one of us to the Physical Society in May, 1885. We fixed two strips of tinfoil, $a a$, of such dimensions that they correspond to the electrified surfaces of one of our most efficient ozone tubes, on the remote sides of sheets of patent plate glass $b b$, which are mounted on a small parallel vice, so that they can be made to recede from, and approach, each other (Fig. 6). The tinfoil is then connected with the wires from the coil, and the character of the discharge between the plates can be examined. When the glass plates are at a distance of a centimetre, the discharge between them consists of a few sparks, not very close together, corresponding in some degree to the spark discharge of the Rhumkorf coil, and it is by no means silent. If the plates are made to approach more closely, the number of these sparks increases till they become very numerous. When the distance approaches more nearly to that which we employ in the ozone generators, viz., $1 \mathrm{~mm}$., we perceive, mixed with these sparks, what we may describe, for want of a better term, as a glow discharge, and if the plates be brought still a little closer, the discharge appears to consist of this glow almost entirely, with only a few of the larger sparks at considerable intervals. If now a little warm moist air be blown between the plates, the glow vanishes, the discharge is once more composed of comparatively few and large sparks. These are gradually replaced by the glow after the lapse of a few minutes. If,

* The results of an unpublished experiment by one of us confirms the idea that peroxide of nitrogen is sometimes formed in ozone generators. 
however, the discharge has been long passing between a pair of plates so that they have become warm, it is more difficult to obtain such startling contrasts, though it is not impossible to effect similar but less complete modifications of the discharge. We have also examined the discharge through oxygen. For this purpose, the plates $b b$ were brought within a distance of $1 \mathrm{~mm}$., and cemented at their edges with solid paraffin. Oxygen was conveyed to the flat vessel so obtained, and allowed to pass between the plates and escape at an opening left for that purpose, which also permitted us to observe the discharge within. The general character of the discharge corresponded with that of the discharge in air, but the glow discharge in oxygen seemed to us to present a somewhat washed-out appearance as compared with the glow discharge in air. From the considerable resemblance between the discharge which occurs between the two dry plates of glass when near to each other, and the glow discharge with which Andrews and Tait obtained such remarkable proportions of ozone from oxygen, and from the tendency of moisture on the plates to produce more or less the larger sparks which are known to destroy ozone when its amount exceeds certain narrow limits, we are inclined to consider that, at least to some extent, the high yield of ozone which appears to us to be produced by very dry oxygen, is due to the condition of the interior surfaces of the apparatus being exceedingly favourable to the occurrence of that form of electric discharge which would seem to be most efficient in effecting the conversion of oxygen into ozone.

In presenting these results, we would explain that we have made preparations for making other experiments of the same kind, more particularly with the object of gaining further information concerning the chemical activity of dry ozone, and concerning the efficiency of ozone generators of the type employed by Andrews and Tait, as compared with those of the Siemens form, which are now so generally employed. A long delay, however, must occur before these can be finished, and as we think the subject is one of considerable interest, we have not hesitated to bring forward by itself that portion of the work which is now completed. 